\title{
Preferencia del consumo de ensilajes de árboles nativos por venados cola blanca (Odocoileus virginianus) en cautiverio
}

\author{
Montes Pérez, R.C. ${ }^{\circledR}$; Ceballos Centeno, S.C. y Solorio Sánchez, F.J.
}

Campus de Ciencias Biológicas y Agropecuarias. Universidad Autónoma de Yucatán. Mérida. Yucatán. México.

\section{PalABRAS CLAVE ADICIONALES}

\section{B. alicastrum}

Fibra detergente neutro.

L. leucocephala.

Proteína bruta.

\section{ADDITIONAL KEYWORDS \\ B. alicastrum. \\ Crude protein. \\ L. leucocephala. \\ Neutral detergent fiber.}

INFORMACIÓN

Cronología del artículo.

Recibido/Received: 4.11.2014

Aceptado/Accepted: 13.5.2015

On-line: 16.9 .2015

Correspondencia a los autores/Contact e-mail:

mperez@uady.mx

\section{RESUMEN}

El objetivo de este trabajo fue evaluar la preferencia de ensilajes de forraje nativo por Odocoileus virginianus. El estudio se efectuó con nueve $O$. virginianus adultos machos y hembras, confinados en tres Unidades de manejo de vida silvestre en Yucatán, México. Se utilizó el diseño de cuadro latino $3 \times 3$ para probar el consumo y preferencia de ensilajes. Los venados cola blanca tuvieron mayor preferencia por ensilaje de Brosimum alicastrum que Leucaena leucocephala y la mezcla de ambos al 50\% ( $p<0,01)$. Hubo correlación negativa entre la cantidad de fibra detergente neutro y el consumo de materia seca en peso metabólico $(p=0,062)$.

\section{Native trees silage consumption by white tailed-deer (Odocoileus virginianus) in captivity}

\section{SUMMARY}

The objective of this work was to evaluate the preference of silages native forage by Odocoileus virginianus. The study was carried out with nine adults deer males and females confined in three management wildlife production units in Yucatan, Mexico. Latin square design $3 \times 3$ was used to test the silages consumption and preference. White-tailed deer had higher preference by silages of Brosimum alicastrum than Leucaena leucocephala and both mixture at $50 \%(p<0.01)$. There was a negative correlation between the amount of neutral detergent fiber and dry matter intake in metabolic weight $(p=0.062)$.

\section{INTRODUCCIÓN}

Los ensilajes son de uso común para la alimentación del ganado; los principales alimentos que se ensilan son maíz, trigo, y cebada entre otros (Emile et al., 2007). Sin embargo en fauna silvestre ha sido poco utilizado. Kim et al. (1996) emplearon ensilados de maíz y centeno para alimentar venados Sika. Rajský et al. (2008) también sometieron a prueba el consumo de ensilados de maíz, pasto y mezclas de éstos en ciervo rojo (Cervus elaphus), pero no se ha reportado el consumo de ensilados de follaje de árboles nativos en venados cola blanca (Odocoileus virginianus).

Se ha reportado que los venados cola blanca en Yucatán, consumen 50 especies vegetales nativas (Sandoval, 1991). Plata et al. (2009) informan los consumos de 11 especies vegetales, entre ellas de Leucaena leucocephala (Lam.) de Wit y Brosimum alicastrum Sw. subsp. alicastrum C.C. Berg (1972), con valores de 0,296 y 0,246 g/kg Materia seca, que corresponde al 28,02 y $23,23 \%$ de su dieta, respectivamente. Castillo et al.
(2009) también reportaron el consumo de materia fresca de B. alicastrum, L. leucoephala, y G. Guazuma ulmifolia, por venados cola blanca, por tanto es de esperarse que si la elaboración de ensilajes con estos forrajes, mantiene los contenidos nutricionales originales de los alimentos frescos, los venados podrían consumirlos. El objetivo de este trabajo es evaluar en $O$. virginianus la preferencia del consumo en materia seca de ensilajes de B. alicastrum, L. leucocephala y mezcla de ambos al $50 \%$.

\section{MATERIAL Y MÉTODOS}

El estudio se realizó en tres unidades de manejo y conservación de vida silvestre (UMA): Xmatkuil (Latitud: $20^{\circ} 51^{\prime} 39,98^{\prime \prime} \mathrm{N}$. Longitud: $89^{\circ} 36^{\prime} 32,28^{\prime \prime} \mathrm{W}$, Elevación: 10,48 msnm). UMA Yuumbaalche il, (Latitud: $20^{\circ} 48^{\prime} 45,12^{\prime \prime}$ N. Longitud: $89^{\circ} 30^{\prime} 22,53^{\prime \prime}$ W. Elevación: $15 \mathrm{msnm}$.). UMA San Fernando, (Latitud: $20^{\circ} 23^{\prime} 44,73^{\prime \prime}$ N. Longitud: 89 31' 14,61' W. Elevación: $26 \mathrm{msnm}$.). Las tres localizadas en el estado de Yucatán, México. El clima es tropical subhúmedo Awo (i)’g (García, 2004). Con estación lluviosa en verano, preci- 
Tabla I. Composición química de los ensilados en \% (Chemical composition of the silage in \%).

\begin{tabular}{lccccccc}
\hline Tratamiento de ensilaje & MS & FDN & FDA & EE & PC & TC & FT \\
\hline A (B. alicastrum) & 40,0 & $51,69^{\mathrm{a}}$ & 43,72 & 2,73 & 14,36 & 0,0 & 0,32 \\
B (L. leucocephala) & 30,3 & $65,55^{\mathrm{b}}$ & 52,02 & 1,93 & 19,63 & 0,0 & 0,22 \\
C (A 50\%, B 50\%) & 29,5 & $60,59^{\mathrm{a}, \mathrm{b}}$ & 54,74 & 1,61 & 16,53 & 0,0 & 0,2
\end{tabular}

$M S=$ materia seca; $F D N=$ fibra detergente neutro; FDA= fibra detergente ácido; $\mathrm{EE}=$ extracto etéreo; $\mathrm{PC}=$ proteína cruda; $\mathrm{TC}=$ taninos condensados; $\mathrm{FT}=$ fenoles totales. Diferente literal en la misma columna indica diferencia significativa $p=0,061$.

Tabla II. Consumo de materia seca con base al peso metabólico del animal por cuatro horas (g/kg PM/4 horas) de cada uno de los ensilados probados en $O$. virginianus (Matter intake dry based on the metabolic weight of the animal for four hours ( $\mathrm{g} / \mathrm{kg} \mathrm{PM} / 4$ hours) of each of the tested or silage in O. virginianus).

\begin{tabular}{lrrrr}
\hline Tratamiento & Media & $\begin{array}{c}\text { Error } \\
\text { estándar }\end{array}$ & $\begin{array}{c}\text { Límite } \\
\text { inferior }\end{array}$ & $\begin{array}{c}\text { Límite } \\
\text { superior }\end{array}$ \\
\hline A (B. alicastrum) & $13,890^{\text {a }}$ & 1,176 & 11,536 & 16,244 \\
B (L. leucocephala) & $2,763^{\text {b }}$ & 1,176 & 0,409 & 5,117 \\
C (A 50\%, B 50\%) & $4,804^{\text {b }}$ & 1,176 & 2,450 & 7,157
\end{tabular}

$\mathrm{C}=$ ensilaje de $B$. alicastrum y $L$. leucocephala $(50 \%, 50 \%)$; $B=$ ensilaje de $L$. leucocephala; $A=$ ensilaje de $B$. alicastrum. Diferente literal en la misma columna indica diferencia significativa $(p<0,01)$.

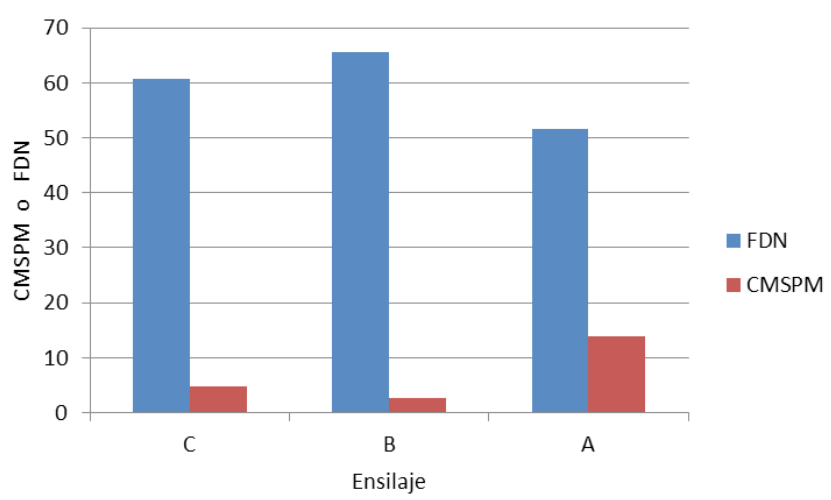

Figura 1. Consumo promedio de materia seca con base al peso metabólico del animal por 4 horas $(\mathrm{g} / \mathrm{kg}$ peso metabólico/4 horas, CMSPM) y contenido en $\%$ de fibra detergente neutro de cada ensilaje (FDN). $C$ es ensilaje de B. alicastrum y L. leucocephala en proporciones iguales, B es ensilaje de L. leucocephala, A es ensilaje de B. alicastrum (Average consumption of matter dry based on the metabolic weight of the animal for 4 hours ( $\mathrm{g} / \mathrm{kg}$ weight metabolico/4 hours, CMSPM) and content in $\%$ neutral detergent fiber of each silage (NDF). $C$ is silage from $B$. alicastrum and $L$. leucocephala in equal proportions, $B$ is silage of $L$. leucocephala, A is silage from $B$. alicastrum).

pitación pluvial anual de 838 a $1128 \mathrm{~mm}$, y temperatura ambiental media anual de 24,5 a $27^{\circ} \mathrm{C}$ (Duch, 1988).

Se sometieron a prueba un total de 9 venados cola blanca (Odocoileus virginianus) adultos, 4 machos y 5 hembras, con pesos vivos promedio (PV) de $40 \pm 3,8 \mathrm{~kg}$. Los animales de la UMA Xmatkuil recibían de alimento papaya (Carica papaya), calabaza (Cucurbita pepo) y pepino (Cucumis sativus) 1,8 kg/animal diariamente. En la UMA Yuumbaalche il, recibían 1,9 kg de forraje fresco de P. purpureum o C. plectostachius, y alimento balanceado para ganado bovino con $12 \%$ proteína cruda (300 g/ animal). En la UMA San Fernando se les proporcionaba forraje picado de B. alicastrum 2,2 kg/animal/día, y agua ad libitum en todas las UMA.

Los forrajes ensilados fueron de B. alicastrum (ramón) y L. leucocephala (huaxin). El proceso de ensilaje se efectuó de acuerdo a lo reportado por Valencia et al. (2011), después de la colecta del forraje, se procedió al picado y mezclado de éste con melaza al $5 \%$ e inmediatamente la compactación. Los silos se almacenaron en bolsas de polietileno tubulares e introducidas en sacos para mayor protección. El tiempo de espera fue de un mes y medio, antes de abrir los silos para ofertar a los venados.

Se prepararon tres tratamientos de ensilajes: A corresponde al tratamiento de ensilado con $95 \%$ B. alicastrum y $5 \%$ de melaza. B corresponde al tratamiento de ensilado con $95 \%$ de L. leucocephala y $5 \%$ melaza. C corresponde al tratamiento de ensilado con $47,5 \%$ B. alicastrum, 47,5\% L. leucocephala y 5\% melaza. Se realizó el análisis químico a cada uno de los tres tratamientos, para determinar FDA, FDN, PC, MS, EE, taninos condensados (TC) y fenoles totales (FT) de acuerdo a la AOAC (1995) y Van Soest et al. (1991).

La primera etapa fue de adaptación, en ésta se les proporcionó ensilados antes de entregarles el alimento diario normal, se ofrecieron los ensilados en tres contenedores ubicados en lugares diferentes por 4 horas durante 10 días consecutivos. Transcurridas las 4 horas, se ofreció el alimento normal diario.

En la prueba de preferencia se les proporcionaron en recipientes separados los tratamientos de ensilajes, distribuidos en tres posiciones diferentes de cada corral, que se cambiaron de posición cada día, la oferta fue ad libitum por 4 horas al día, durante un periodo de 3 días, de acuerdo al cuadro latino $3 \times 3$. Este diseño se replicó al menos 2 veces en cada una de las UMAs. Se pesaron los silos antes y después de proporcionarlos, para estimar con base en el peso metabólico la cantidad consumida individual (CMSPM) en base seca. Las diferencias de los consumos de alimento de los tres tratamientos de forraje ensilado, se evaluó mediante la prueba de análisis de varianza con doble restricción del cuadro latino $3 \times 3$. Se efectuó el análisis de correlación entre las cantidades de compuestos nutricionales (PC, EE, TC, FT, FDN, FDA) y el CMSPM para cada uno de los tratamientos. Se contrastaron los valores de FDN, FDA, PC y MS entre los tratamientos, mediante Chi cuadrada.

\section{RESULTADOS Y DISCUSIÓN}

En la tabla I se muestran los resultados del análisis químico proximal de cada uno de los ensilajes probados. El pH de los silos varió de 4,2 a 5,0. En todos los casos el color fue verde olivo, el aroma fue agridulce, la textura fibrosa y ligeramente pastosa. La tabla II muestra la comparación múltiple de medias entre cada tratamiento. La figura 1 muestra la relación inversa entre los CMSPM de cada ensilaje respecto a la fibra detergente neutra FDN, resultando un coeficiente de 
correlación $r=-0,981(p<0,062)$. Los porcentajes de MS de los ensilajes utilizados fue de 29,54 a $40 \%$ (sin presentar diferencia significativa, $p>0,05)$. Sin embargo se observa un ligero aumento de MS en el ensilaje de $B$. alicastrum, mientras que el ensilaje de la combinación de ambos, B. alicastrum y L. leucocephala fue ligeramente menor, posiblemente por el uso de material vegetal un poco más tierno utilizado en la elaboración del silo.

El consumo de MS por venados se ha reportado en $4 \%$ de su PV (Sánchez, 2011), los ejemplares sometidos a prueba tuvieron peso promedio de $40 \mathrm{~kg}$, resultaría un consumo diario de 1,6 kg de MS de ensilado por el venado cola blanca. En el estudio de Lizárraga et al. (2001), la MS de las hojas y tallos del forraje fresco de $B$. alicastrum (41,8 y 44,5\% MS respectivamente) fue similar, pero ligeramente mayor a la obtenida del ensilaje, y para L. leucocephala, en el mismo trabajo, los valores de MS en hojas y tallos fue de 34,9\% y 33,3\% respectivamente, ligeramente mayores al encontrado en el ensilaje (30,3\%). Riaño et al. (2004) reportan que los cambios del valor de MS en una planta, está influido por el tiempo transcurrido, la exposición a altas temperaturas y la irradiación solar acumulados, conforme estos factores aumentan, la materia seca de la planta también se acumula. Durante la primera etapa de crecimiento la materia seca se distribuye principalmente en las hojas, la raíz y el tallo, presentando al final acumulación en las ramas, a expensas de una disminución en la raíz y las hojas, esto explica la variación de MS dentro de la misma planta en sus hojas y tallos.

El contenido nutricional de los ensilados mostró que el mayor contenido de PC fue para el ensilado de L. leucocephala, y el menor fue para B. alicastrum, sin presentar diferencia significativa $(p>0,05)$. De acuerdo a Sosa et al. (2004) el valor de PC en ensilaje de $B$. alicastrum, fue ligeramente más alto, pero la PC en $L$. leucocephala ensilada fue menor que el reportado por estos autores, el resultado puede ser debido al nivel de lignificación de las hojas, puesto que se ha reportado que hay una relación inversa entre edad de la planta y grado de lignificación de la materia vegetal, conforme avanza la madurez de la planta el nitrógeno se trasloca de las hojas a los tejidos de reserva, ocasionando disminución del valor proteico y digestibilidad del follaje (Pirela, 2005). El contenido de FDN de los ensilados, fue mayor en el tratamiento B y menor en el tratamiento $A(p=0,061)$. Los valores de FDA fueron mayores para tratamiento $\mathrm{C}$ y menores para Tratamiento $A$ aunque no se observaron diferencias significativas ( $p>0,05)$. Considerando las preferencias de consumo del venado, conocido por su capacidad de seleccionar los forrajes que aporten menor gasto energético por digestión y sustratos con mayor digestibilidad, entonces explica el mayor consumo de ensilaje del B. alicastrum, debido al bajo nivel de material lignocelulósico.

No se detectó contenido de TC en ensilajes de $L$. leucocephala y B. alicastrum. Castillo et al. (2009) reportaron valores de 1,9 a 9,1 g/ kg en forraje fresco de estas dos especies, colectadas en la misma localidad de este trabajo. Por otra parte los valores de FT para B. alicastrum y L. leucocephala frescos en el trabajo de Castillo et al. (2009) es de 1,1\%, mayores al registrado en este momento, información que corresponde a forraje colec- tado en la misma localidad de este trabajo; por tanto es posible proponer la hipótesis de que el proceso de ensilado disminuye los compuestos TC y FT, lo cual está de acuerdo a lo informado por Mendoza y Martínez (2010), que mencionan una pérdida de 1 a $2 \%$ en los nutrimentos de forraje ensilado, cuando el método se aplica inmediatamente al corte. Por otra parte Rugna et al. (2008) reportaron que en Smilax campestris el valor de FT y TC varía de acuerdo a la edad, los FT que producen las hojas jóvenes es 15\% mayor que en las hojas adultas, la concentración de TC en hojas jóvenes es superior al de hojas adultas en 65,6\%. Es una estrategia de protección contra la herbivoría porque los compuestos secundarios pueden alterar la palatabilidad de las plantas (Launchbaugh, 1996; Kimball y Nolte, 2005). Es posible que el ensilaje de $B$. alicastrum contenía principalmente hojas adultas, y además elevada MS, rasgo que está relacionado de manera directa con el aumento de consumo de materia seca en rumiantes (Pendini, 2009), y a pesar del relativo bajo valor de PC, los venados prefirieron consumir este ensilaje $(p<0,05)$ sobre los otros; sin embargo, el nivel de PC en B. alicastrum, es suficiente para cubrir sus requerimientos en crecimiento, gestación y lactancia (Plata et al., 2009).

Existe una correlación negativa significativa entre FDN y CMSPM $(p<0,062)$, por tanto los venados cola blanca prefieren consumir ensilajes con menor cantidad de FDN. Esto coincide con los resultados de un metanálisis realizado con más de 120 estudios publicados, confirman la relación negativa entre el contenido de FDN y la ingestión de materia seca (IMS) (Bach y Calsamiglia, 2006). Este hallazgo es de esperarse porque Pendini (2009), reporta que el retículo rumen y probablemente el abomaso poseen a lo largo y ancho de sus paredes receptores que afectan negativamente el CMS en relación al volumen y peso del alimento retenido en ellos. La fracción de FDN del alimento, por su baja tasa de digestión, es el principal factor asociado al efecto de llenado del rumen o regulación física del consumo.

En conclusión, los venados cola blanca mostraron preferencia por los ensilados de B. alicastrum sobre L. leucocephala y la mezcla de ambos en proporciones iguales. Está correlacionada negativamente la cantidad de FDN con el CMSPM.

\section{AGRADECIMIENTOS}

Los autores agradecen al Centro de Investigación y Experimentación de Alternativas Agroecológicas S.C., el financiamiento para ejecutar este trabajo de investigación. También expresamos nuestro reconocimiento a los representantes legales y responsables técnicos de las Unidades de Manejo y Conservación de Vida Silvestre Yuumbaalche il y San Fernando, por brindar el acceso a sus instalaciones para ejecutar este proyecto.

\section{BIBLIOGRAFÍA}

AOAC, Association of Official Analytical Chemists. 1995. Methods of Analysis. Washington D.C. USA.

Bach, A. y Calsamiglia, S. 2006. La fibra en los rumiantes ¿Química o Física? Grupo de lnvestigación en Nutrición, Manejoy Bienestar Animal. 
XXII Curso de Especialización FEDNA. IRTA Unidad de Rumiantes, Universidad Autónoma de Barcelona. pp. 99-113. www.produccionanimal.com.ar/informacion_tecnica/manejo_del_alimento/100fibra_en_rumiantes.pdf (22/04/2013).

Castillo, L.F.I.; Montes, P.R.C. y Rodriguez, M.C.E. 2009. Preferencias de consumo de cuatro forrajes por venado cola blanca (Odocoileus virginianus)mantenidos en cautiverio en Yucatán. Cienc Agr, 7: 75-82.

Duch, G.J. 1988. La conformación territorial del estado de Yucatán. Centro Regional de la Península de Yucatán. U. Auton. Chapingo, México. pp: 188-203.

Emile, J.C.; Jobim, C.C.; Surault, F. and Barriére, Y. 2007. Genetic variation in the digestibility in sheep of selected whole-crop cereals used as silages. Animal, 1: 1122-1125.

García E. 2004. Modificaciones al sistema de clasificación climática de Köppen. Serie Libros $n^{\circ}$ 6. Instituto de Geografía. Universidad Nacional Autónoma de México.

Kim, K.H.; Jeon, B.T.; Kim, Y.C.; Kyung, B.H. and Kim, C.W. 1996. A comparison of oak browse (Quercus spp.) and silages of rye and maize with respect to voluntary intake, digestibility, nitrogen balance and rumination time in penned Korean sika deer. Anim Feed Sci Tech, 61: 351-359.

Kimball, B.A. and Nolte, D.L. 2005. Herbivore experience with plant defense compounds influences acquisition of new flavor aversion. Appl Anim Behav Sci, 91: 17-34.

Launchbaugh K.L. 1996. Biochemical aspects of grazing behavior. In: Eds Hodgson J. and Illius A. The ecology and management of grazing systems. CAB International. Wallingford, UK. pp: 159-184.

Lizárraga, S.H.L.; Solorio, S.F.J. y Sandoval, C.C.A. 2001. Evaluación agronómica de especies arbóreas para la producción de forraje en la Península de Yucatán. Livest Res Rural Dev, 13. www.lrrd.org/ Irrd13/6/liza136.htm (03/03/2014).

Mendoza, E.M.V.y Martínez, O.I.A. 2010. Elaboración de ensilaje líquido a base de yuca Manihot sculenta crantz, papa Solanum tuberosum y apio Arracacia xanthorrhiza, como una alternativa de alimentación en la época de verano para ovinos y caprinos del Centro de Investigación Pecuario Guatiguara. http://es.scribd.com/doc/36867018/ArticuloCientifico-Cica-Ensilaje-Liquido-Vers-2\#scribd (19/06/2013).

Pendini, C.R. 2009. Consumo. Primer Curso de la Red ICAARG. Resumen del capítulo alimentación de la vaca lechera. Libro de notas sobre alimentación de la vaca lechera. Ed. SIMA. Córdoba. Argentina. Sistemas de producción lechera de Argentina y Cuba. www.ebah. com.br/content/ABAAAfagcAJ/consumo (06/08/2015).

Pirela, M.F. 2005. Valor nutritivo de los pastos tropicales. Manual de ganadería de doble propósito. Instituto Nacional de Investigaciones Agrícolas. Venezuela. http://www.avpa.ula.ve/docuPDFs/libros_online/manual-ganaderia/seccion3/articulo6-s3.pdf (18/02/2014).

Plata, F.X.; Ebergeny, S.; Reséndiz, J.L.; Villarreal, O.; Bárcena, R.; Viccon, J.A. y Mendoza, G.D. 2009. Palatabilidad y composición química de alimentos consumidos en cautiverio por el venado cola blanca de Yucatán (Odocoileus virginianus yucatanensis). Arch Med Vet, 41: 123-129.

Rajský, M.; Vodñanský, M.; Hell, P.; Slamecka, J.; Kropil, R. and Rajský, D. 2008. Influence supplementary feeding on bark browsing by red deer (Cervus elaphus) under experimental conditions. Eur J Wildlife Res, 54: 701-708.

Riaño, H.N.M.; Arcila, P.J.; Jaramillo, R.A. y Chaves, C.B. 2004. Acumulación de materia seca y extracción d enutrimentos por Coffea arabica L. cv. Colombia en tres localidades de la zona cafetera central. Cenicafé, 55: 265-276.

Rugna, A.; Ricco, R.; Gurni, A. y Wagner, M. 2008. Variación en el contenido de polifenoles foliares en Smilax campestris Griseb Smilacaceae según grado de desarrollo. Lat Am J Pharm, 27: 247-249.

Sánchez, C.B. 2011. Plan de manejo de venado cola blanca (Odocoileus virginianus mexicanus) en la comunidad de Aguacatitla, Hgo. Chapingo Texcoco, Estado de México. http://chapingo.mx/dicifo/ tesislic/2011/sanchez_carrillo_berenice_2011.pdf (23/09/2013).

Sandoval, C.C.A. 1991. El venado cola blanca: selección de los componentes de su dieta en estado silvestre. Rev U Auton Yucatán, 176: 65-68.

Sosa, R.E.E.; Pérez, R.D.; Ortega, R.L. y Zapata, B.G. 2004. Evaluación del potencial forrajero de árboles y arbustos tropicales para la alimentación de ovinos. Tec Pecu Méx, 42: 129-144.

Valencia, C.A.; Hernández, B.A. y López de Buen, L. 2011 . El ensilaje: ¿qué es y para qué sirve? Rev Divul Cient Tecnol U Veracruzana, volumen XXIV número 2. Veracruz, México. http://www.uv.mx/cienciahombre/revistae/vol24num2/articulos/ensilaje/ (03/10/2013).

Van Soest, P.J.; Robertson J. and Lewis B. 1991. Methods for dietary fiber, neutral detergent fiber, and nonstarch polysaccharides in relation to animal nutrition. J Dairy Sci, 74: 3583-3597. 